\title{
Isolation of Peridinin-Related Norcarotenoids with Cell Growth- Inhibitory Activity from the Cultured Dinoflagellate of Symbiodinium sp., a Symbiont of the Okinawan Soft Coral Clavularia viridis, and Analysis of Fatty Acids of the Dinoflagellate
}

\author{
Motoya Suzuki, ${ }^{a}$ Kinzo Watanabe, ${ }^{a}$ Shoko Fujiwara, ${ }^{a}$ Toshie Kurasawa, ${ }^{a}$ Takako WaKabayashi, ${ }^{a}$ \\ Mikio Tsuzuki, ${ }^{a}$ Kazuo IGUCHI, ${ }^{*, a}$ and Takao YAMORI ${ }^{b}$ \\ ${ }^{a}$ School of Life Science, Tokyo University of Pharmacy and Life Science; Horinouchi, Hachioji, Tokyo 192-0392, Japan: \\ and ${ }^{b}$ Cancer Chemotherapy Center, Japanese Foundation for Cancer Research; Kami-ikebukuro, Toshima-ku, Tokyo \\ 170-8455, Japan. $\quad$ Received December 12, 2002; accepted February 27, 2003
}

Two norcarotenoids, 1 and 2 , related to peridinin (3) were isolated from the cultured dinoflagellate of the genus Symbiodinium, a symbiont of the Okinawan soft coral Clavularia viridis, which contains in abundance antitumor marine prostanoids such as clavulones. The structures of 1 and 2 were elucidated on the basis of spectroscopic analysis. These compounds showed significant growth-inhibitory activity in vitro toward cancer cells. Analysis of fatty acids of the dinoflagellate was also carried out, suggesting that the marine prostanoids are produced by the host soft coral itself.

Key words dinoflagellate; Symbiodinium sp.; carotenoid; growth-inhibitory activity; symbiotic alga; fatty acid

The Okinawan soft coral Clavularia viridis has been recognized as a rich source for marine prostanoids, such as clavulones $^{1,2)}$ and chlorovulones. ${ }^{3,4)}$ These marine prostanoids have received much attention owing to their unique structures and remarkable biological activities. ${ }^{1,3,5,6)}$ Biosynthesis of clavulones was also of interest, and was studied by Corey's group, which proposed a unique biosynthetic pathway, ${ }^{7-10)}$ essentially different from that for mammalian prostaglandins. However, the organism producing the marine prostanoids (the host soft coral or the symbiotic alga) has not yet been clarified. This fundamental and interesting question led us to investigate chemical constituents involving fatty acids of the symbiotic alga isolated from the host soft coral, C. viridis, resulting in the isolation of two peridinin-related norcarotenoids. This paper describes the isolation, structures and biological activity of these norcarotenoids, together with results of the analysis of fatty acids of the alga.

The symbiotic alga isolated from $C$. viridis was identified as a dinoflagellate of the genus Symbiodinium by DNA analysis, and was cultured at $23{ }^{\circ} \mathrm{C}$ for $40 \mathrm{~d}$. The cultured alga was extracted with $\mathrm{MeOH}$, and the $\mathrm{MeOH}$ extract (3.66 g) was partitioned between EtOAc and $\mathrm{H}_{2} \mathrm{O}$. The EtOAc-soluble portion (1.48 g) was chromatographed on a silica gel column to give five fractions (fractions $1-5$ ). A portion of fraction $3(116 \mathrm{mg})$ was further chromatographed repeatedly to give compounds 1 ( $2.4 \mathrm{mg})$ and $\mathbf{2}(3.8 \mathrm{mg})$, along with peridinin $^{11-13)}(3,2.2 \mathrm{mg})$. A portion of fraction 1 $\left(78 \mathrm{mg}\right.$ ) was treated with $\mathrm{HCl}-\mathrm{MeOH}$ at $90^{\circ} \mathrm{C}$ for $2 \mathrm{~h}$ to give a mixture of fatty acid methyl esters; this was analyzed by gas chromatography (GC).

The molecular formula of compound $\mathbf{1}$ a reddish viscous oil, $\left.[\alpha]_{\mathrm{D}}^{25}+63.6^{\circ}\left(\mathrm{CHCl}_{3}\right)\right\}$ was found to be $\mathrm{C}_{39} \mathrm{H}_{50} \mathrm{O}_{7}$ by high-resolution electron impact mass spectra (HR-EI-MS). The degree of unsaturation (fifteen) was given from the molecular formula. All 39 carbons $^{14)}$ appeared in the ${ }^{13} \mathrm{C}$-NMR spectrum of 1 (Table 1). The distortionless enhancement by polarization transfer (DEPT) spectrum showed nine methyls, four $s p^{3}$ methylenes, three $s p^{3}$ oxymethines, ten $s p^{2}$ methines, four $s p^{3}$ quaternary carbons, eight $s p^{2}$ quaternary carbons involving two carbonyls, and one $s p$ quaternary carbon. These spectral data, coupled with the reddish color and absorption maximum at $449 \mathrm{~nm}$ ( $\varepsilon 57800)$, suggested that compound 1 has a conjugated polyene system like carotenoids. The IR spectrum showed the presence of hydroxy $\left(3458 \mathrm{~cm}^{-1}\right)$ and acetoxy $\left(1732,1250 \mathrm{~cm}^{-1}\right)$ groups. The presence of an allene group was indicated by the IR absorption at $1929 \mathrm{~cm}^{-1}$ and by the low-field ${ }^{13} \mathrm{C}$-NMR signal at $\delta 202.6$ (C, C-7') ppm. The ${ }^{1} \mathrm{H}-\mathrm{NMR}$ spectrum of $\mathbf{1}$ disclosed three oxymethine protons at $\delta 4.24(1 \mathrm{H}$, quint., $J=3.9 \mathrm{~Hz}, \mathrm{H}-3), 5.38(1 \mathrm{H}, \mathrm{tt}$, $\left.J=12.1,4.1 \mathrm{~Hz}, \mathrm{H}-3^{\prime}\right)$ and $5.61(1 \mathrm{H}$, br s, H-8) ppm, ten olefinic protons, eight methyl protons, and one acetoxy methyl proton at $\delta 2.04(3 \mathrm{H}, \mathrm{s})$, as shown in Table 1. The ${ }^{13} \mathrm{C}$-NMR signals at $\delta 168.9$ (C) and 170.4 (C) ppm showed the presence of two ester groups, one of which was attributed to the above-mentioned acetoxy group. These spectral data resembled those of peridinin (3) (see Experimental), except for the NMR signals due to the 5, 6, 7 and 8 positions.

After assignments of the $\mathrm{C}-\mathrm{H}$ direct bondings were made based on heteronuclear multiple-quantum coherence (HMQC), the heteronuclear multiple-bond correlation (HMBC) spectrum was measured. Analysis of $\mathrm{C}-\mathrm{H}$ long-
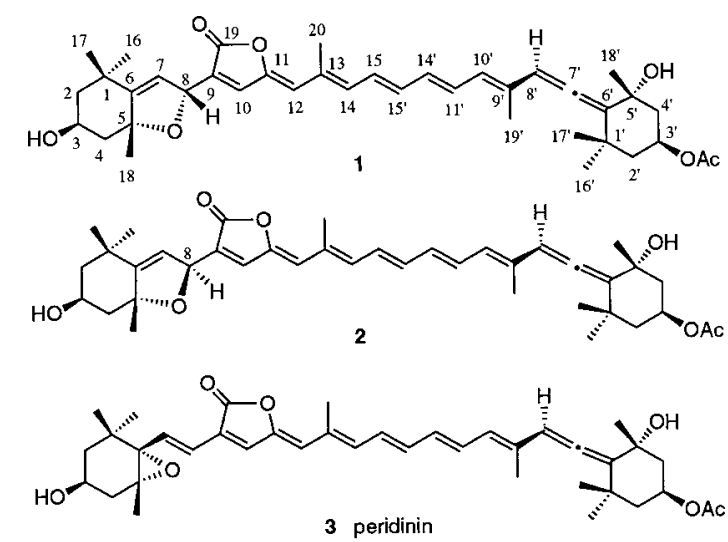
range correlations (two or three bonds) in the HMBC spectrum, as shown by dotted lines in Fig. 1, led to the gross structure for $\mathbf{1}$. Checking the resultant structure of $\mathbf{1}$ through references, compounds $\mathbf{1}$ and $\mathbf{2}$ were found. ${ }^{15,16)}$ These compounds were not natural products, but products obtained from peridinin (3) by treating 3 with dilute $\mathrm{HCl}$ in $\mathrm{MeOH}$
$(0.03 \mathrm{M})$ at room temperature. These compounds were found to be diastereomeric at $\mathrm{C}-8$ on the dihydrofuran ring, and the stereochemistry at C-8 was determined based on NOE analysis. ${ }^{16)}$ The spectral data of the present 1 were identical with those reported. ${ }^{16)}$ Compound $\mathbf{1}$ was thus determined to be alltrans- $\left(8 R, 6^{\prime} R\right)$-peridinin-5,8-furanoxide.

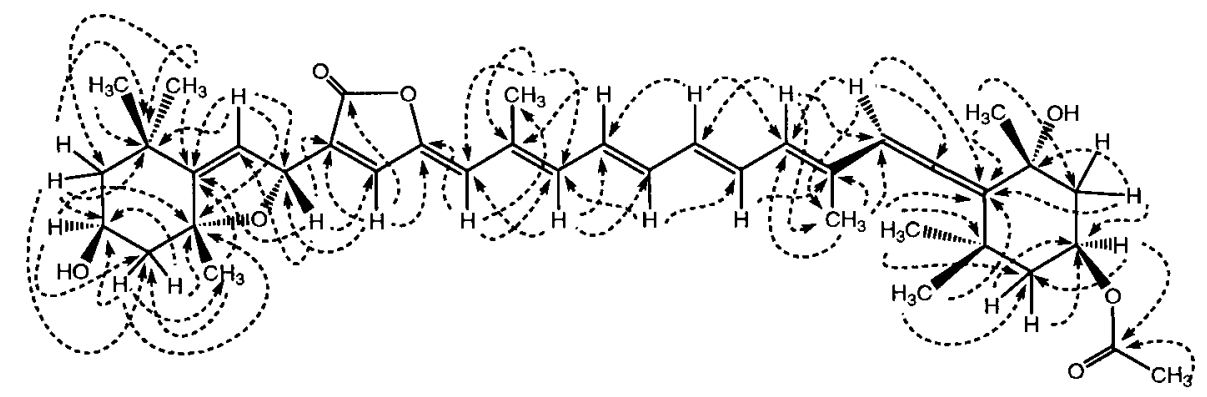

Fig. 1. HMBC Correlations for $\mathbf{1}$

Table 1. NMR Data for $\mathbf{1}$ and $\mathbf{2}^{a}$

\begin{tabular}{|c|c|c|c|c|}
\hline \multirow{2}{*}{ No. } & \multicolumn{2}{|c|}{1} & \multicolumn{2}{|c|}{2} \\
\hline & ${ }^{13} \mathrm{C}(125 \mathrm{MHz})^{b)}$ & ${ }^{1} \mathrm{H}(500 \mathrm{MHz}){ }^{c)}$ & ${ }^{13} \mathrm{C}(125 \mathrm{MHz}){ }^{b)}$ & ${ }^{1} \mathrm{H}(500 \mathrm{MHz})^{c)}$ \\
\hline 1 & $33.9(\mathrm{C})$ & & $34.2(\mathrm{C})$ & \\
\hline \multirow[t]{2}{*}{2} & $46.6\left(\mathrm{CH}_{2}\right)$ & $1.49(\mathrm{dd}, 14.3,3.9)$ & $47.1\left(\mathrm{CH}_{2}\right)$ & $1.49(\mathrm{dd}, 14.3,3.7)$ \\
\hline & & $1.76(\mathrm{ddd}, 14.3,3.9,1.4)$ & & $1.79(\mathrm{~m})$ \\
\hline 3 & $67.7(\mathrm{CH})$ & 4.24 (quint, 3.9) & $67.8(\mathrm{CH})$ & 4.28 (quint, 3.7) \\
\hline \multirow[t]{2}{*}{4} & $47.5\left(\mathrm{CH}_{2}\right)$ & $1.94(\mathrm{dd}, 13.6,3.9)$ & $47.0\left(\mathrm{CH}_{2}\right)$ & $1.93(\mathrm{dd}, 13.8,3.7)$ \\
\hline & & $2.18(\mathrm{brdd}, 13.6,3.9)$ & & $2.19(\mathrm{~m})$ \\
\hline 5 & $87.8(\mathrm{C})$ & & $87.9(\mathrm{C})$ & \\
\hline 6 & $153.8(\mathrm{C})$ & & $153.3(\mathrm{C})$ & \\
\hline 7 & $117.8(\mathrm{CH})$ & $5.53(\mathrm{~d}, 1.2)$ & $117.0(\mathrm{CH})$ & $5.64(\mathrm{~d}, 2.1)$ \\
\hline 8 & $77.2(\mathrm{CH})$ & $5.61(\mathrm{brs})$ & $78.1(\mathrm{CH})$ & $5.51(\mathrm{brs})$ \\
\hline 9 & $132.4(\mathrm{C})$ & & $133.2(\mathrm{C})$ & \\
\hline 10 & $138.2(\mathrm{CH})$ & $7.16(\mathrm{~d}, 1.3)$ & $137.5(\mathrm{CH})$ & $7.19(\mathrm{~d}, 1.4)$ \\
\hline 11 & $146.7(\mathrm{C})$ & & $146.8(\mathrm{C})$ & \\
\hline 12 & $118.8(\mathrm{CH})$ & $5.69(\mathrm{~s})$ & $118.7(\mathrm{CH})$ & $5.72(\mathrm{~s})$ \\
\hline 13 & $133.8(\mathrm{C})$ & & $133.7(\mathrm{C})^{d)}$ & \\
\hline 14 & $137.8(\mathrm{CH})$ & $6.42(\mathrm{brd}, 11.6)$ & $137.7(\mathrm{CH})$ & $6.43(\mathrm{brd}, 11.5)$ \\
\hline 15 & $128.8(\mathrm{CH})$ & $6.60(\mathrm{dd}, 11.6,14.2)$ & $128.8(\mathrm{CH})$ & $6.58(\mathrm{~m})$ \\
\hline 16 & $28.6\left(\mathrm{CH}_{3}\right)$ & $1.34(\mathrm{~s})$ & $28.1\left(\mathrm{CH}_{3}\right)$ & $1.32(\mathrm{~s})$ \\
\hline 17 & $31.3\left(\mathrm{CH}_{3}\right)$ & $1.16(\mathrm{~s})$ & $31.3\left(\mathrm{CH}_{3}\right)$ & $1.20(\mathrm{~s})$ \\
\hline 18 & $28.7\left(\mathrm{CH}_{3}\right)$ & $1.66(\mathrm{~s})$ & $31.0\left(\mathrm{CH}_{3}\right)$ & $1.68(\mathrm{~s})$ \\
\hline 19 & $168.9(\mathrm{C})$ & & $169.0(\mathrm{C})$ & \\
\hline 20 & $15.4\left(\mathrm{CH}_{3}\right)$ & $2.21(\mathrm{~s})$ & $15.4\left(\mathrm{CH}_{3}\right)$ & $2.21(\mathrm{~s})$ \\
\hline $1^{\prime}$ & $35.8(\mathrm{C})$ & & $35.8(\mathrm{C})$ & \\
\hline \multirow[t]{2}{*}{$2^{\prime}$} & $45.4\left(\mathrm{CH}_{2}\right)$ & $1.40(\mathrm{t}, 12.1)$ & $45.4\left(\mathrm{CH}_{2}\right)$ & $1.40(\mathrm{t}, 12.0)$ \\
\hline & & $1.99(\mathrm{ddd}, 12.1,4.1,2.0)$ & & $1.99(\mathrm{ddd}, 12.0,4.1,1.9)$ \\
\hline $3^{\prime}$ & $67.9(\mathrm{CH})$ & $5.38(\mathrm{tt}, 12.1,4.1)$ & $67.9(\mathrm{CH})$ & $5.38(\mathrm{tt}, 12.0,4.1)$ \\
\hline \multirow[t]{2}{*}{$4^{\prime}$} & $45.2\left(\mathrm{CH}_{2}\right)$ & $1.51(\mathrm{dd}, 12.8,12.1)$ & $45.2\left(\mathrm{CH}_{2}\right)$ & $1.50(\mathrm{~m})$ \\
\hline & & $2.28(\mathrm{ddd}, 12.8,4.1,2.0)$ & & 2.28 (ddd, $12.9,4.1,1.9)$ \\
\hline $5^{\prime}$ & $72.7(\mathrm{C})$ & & $72.7(\mathrm{C})$ & \\
\hline $6^{\prime}$ & $117.6(\mathrm{C})$ & & $117.6(\mathrm{C})$ & \\
\hline $7^{\prime}$ & $202.6(\mathrm{C})$ & & $202.6(\mathrm{C})$ & \\
\hline $8^{\prime}$ & $103.3(\mathrm{CH})$ & $6.05(\mathrm{~s})$ & $103.3(\mathrm{CH})$ & $6.05(\mathrm{~s})$ \\
\hline $9^{\prime}$ & $133.6(\mathrm{C})$ & & $133.6(\mathrm{C})^{d)}$ & \\
\hline $10^{\prime}$ & $128.1(\mathrm{CH})$ & $6.10(\mathrm{brd}, 11.8)$ & $128.1(\mathrm{CH})$ & $6.11(\mathrm{brd}, 11.5)$ \\
\hline $11^{\prime}$ & $131.4(\mathrm{CH})$ & $6.61(\mathrm{dd}, 14.5,11.8)$ & $131.1(\mathrm{CH})$ & $6.60(\mathrm{~m})$ \\
\hline $14^{\prime}$ & $133.0(\mathrm{CH})$ & $6.37(\mathrm{dd}, 14.5,11.0)$ & $133.0(\mathrm{CH})$ & $6.37(\mathrm{dd}, 14.4,11.0)$ \\
\hline $15^{\prime}$ & $137.1(\mathrm{CH})$ & $6.50(\mathrm{dd}, 14.2,11.0)$ & $137.0(\mathrm{CH})$ & $6.50(\mathrm{dd}, 14.3,11.0)$ \\
\hline $16^{\prime}$ & $31.3\left(\mathrm{CH}_{3}\right)$ & $1.35(\mathrm{~s})$ & $31.3\left(\mathrm{CH}_{3}\right)$ & $1.35(\mathrm{~s})$ \\
\hline $17^{\prime}$ & $32.1\left(\mathrm{CH}_{3}\right)$ & $1.07(\mathrm{~s})$ & $32.0\left(\mathrm{CH}_{3}\right)$ & $1.07(\mathrm{~s})$ \\
\hline $18^{\prime}$ & $29.2\left(\mathrm{CH}_{3}\right)$ & $1.38(\mathrm{~s})$ & $29.2\left(\mathrm{CH}_{3}\right)$ & $1.38(\mathrm{~s})$ \\
\hline $19^{\prime}$ & $14.0\left(\mathrm{CH}_{3}\right)$ & $1.80(\mathrm{~s})$ & $14.0\left(\mathrm{CH}_{3}\right)$ & $1.80(\mathrm{~s})$ \\
\hline $\mathrm{COCH}_{3}$ & $21.4\left(\mathrm{CH}_{3}\right)$ & $2.04(\mathrm{~s})$ & $21.4\left(\mathrm{CH}_{3}\right)$ & $2.04(\mathrm{~s})$ \\
\hline $\mathrm{COCH}_{3}$ & $170.4(\mathrm{C})$ & & $170.4(\mathrm{C})$ & \\
\hline
\end{tabular}

a) Assignments of the ${ }^{13} \mathrm{C}$ and ${ }^{1} \mathrm{H}$ signals were made based on $\mathrm{HMQC}$ analysis. b) $\delta$ ppm in $\mathrm{CDCl}_{3}$. c) $\delta$ ppm in $\mathrm{CDCl}_{3}, J$ in $\mathrm{Hz}$. d) Values with the subscript may be interchanged. 
The molecular formula of compound $\mathbf{2}$ a reddish viscous oil, $\left.[\alpha]_{\mathrm{D}}^{25}-69.2^{\circ}\left(\mathrm{CHCl}_{3}\right)\right\}$ was found to be $\mathrm{C}_{39} \mathrm{H}_{50} \mathrm{O}_{7}$, the same as for 1. NMR data of $\mathbf{2}$ were almost superimposable on those of $\mathbf{1}$ except for the signals around the chiral center at C-8, as shown in Table 1 . These findings strongly suggested that compound $\mathbf{2}$ is a diastereomer of $\mathbf{1}$ at $\mathrm{C}-8$. The spectral data of 2 were shown to be identical with those of $\mathbf{2}^{16)}$ obtained from peridinin (3). Compound 2 was thus determined to be all-trans-( $\left.8 S, 6^{\prime} R\right)$-peridinin-5,8-furanoxide. ${ }^{17)}$

Peridinin and related compounds were known to exhibit anti-tumor and anti-carcinogenic activities. ${ }^{18-20)}$ Therefore, compounds $\mathbf{1}$ and $\mathbf{2}$ were examined for growth-inhibitory activities in vitro toward human cancer cells, as evaluated in the Japanese Foundation for Cancer Res. 39 cell line assay, ${ }^{21)}$ and the results are summarized in Table 2. Compound 2 showed moderate activities at $\mathrm{IC}_{50} 2.0-2.8 \mu \mathrm{g} / \mathrm{ml}$ against BSY-1 (breast cancer), SNB-75 [central nervous system (CNS) cancer], HCT-116 (colon cancer), NCI-H522 (lung cancer), DMS114 (lung cancer) and MKN7 (stomach cancer) cells. Interestingly, the activities of $\mathbf{2}$ were stronger than those of the diastereomeric compound $\mathbf{1}$. The pattern of differential growth-inhibition for $\mathbf{2}$ was evaluated by the Compare Program, and was revealed not to be correlated with that shown by any of the other compounds, including currently used anticancer drugs; the correlation coefficient value was less than 0.5 . This indicates that $\mathbf{2}$ may have a new mode of action.

The mixture of fatty acid methyl esters was analyzed by GC: methyl esters were assigned by comparison of their retention times with those of authentic samples. The main fatty

Table 2. Growth-Inhibitory Activity for $\mathbf{1}$ and $\mathbf{2}$ against 39 Human Cancer Cell Lines

\begin{tabular}{|c|c|c|c|c|c|}
\hline \multirow{2}{*}{ Panel/Cell line } & \multicolumn{2}{|c|}{$\mathrm{IC}_{50}(\mu \mathrm{g} / \mathrm{ml})^{a)}$} & \multirow{2}{*}{ Panel/Cell line } & \multicolumn{2}{|c|}{$\mathrm{IC}_{50}(\mu \mathrm{g} / \mathrm{ml})$} \\
\hline & 1 & 2 & & 1 & 2 \\
\hline Breast cancer & & & Melanoma & & \\
\hline HBC-4 & $-^{b)}$ & - & LOX-IMVI & - & 6.3 \\
\hline BSY-1 & 4.3 & 2.6 & Ovarian cancer & & \\
\hline HBC-5 & - & 8.2 & OVCAR-3 & 6.9 & 4.5 \\
\hline MCF-7 & 9.5 & 3.7 & OVCAR-4 & - & 8.2 \\
\hline MDA-MB-231 & 9.5 & 8.2 & OVCAR-5 & - & - \\
\hline CNS cancer $^{c)}$ & & & OVCAR-8 & - & 3.9 \\
\hline U251 & - & 7.6 & SK-OV-3 & - & - \\
\hline SF-268 & - & 7.6 & Renal cancer & & \\
\hline SF-295 & - & 6.3 & RXF-631L & - & - \\
\hline SF-539 & - & 8.2 & ACHN & - & - \\
\hline SNB-75 & - & 2.2 & Stomach cancer & & \\
\hline SNB-78 & - & 8.2 & St-4 & - & 7.6 \\
\hline Colon cancer & & & MKN1 & 6.9 & 7.6 \\
\hline HCC2998 & - & 8.8 & MKN7 & 5.0 & 2.8 \\
\hline KM-12 & - & 8.2 & MKN28 & - & - \\
\hline HT-29 & - & - & MKN45 & - & 8.2 \\
\hline HCT-15 & - & 8.2 & MKN74 & 4.4 & 3.0 \\
\hline HCT-116 & 4.2 & 2.8 & Prostate cancer & & \\
\hline Lung cancer & & & DU-145 & - & - \\
\hline NCI-H23 & - & 5.2 & PC-3 & 9.5 & - \\
\hline NCI-H226 & - & 3.6 & & & \\
\hline NCI-H522 & 5.4 & 2.3 & & & \\
\hline NCI-H460 & - & - & & & \\
\hline A549 & - & 5.9 & & & \\
\hline DMS273 & 7.6 & 6.2 & & & \\
\hline DMS114 & 3.3 & 2.0 & & & \\
\hline
\end{tabular}

a) Each $\mathrm{IC}_{50}$ value was estimated from its $\mathrm{GI}_{50}$ value, the concentration that yielded $50 \%$ growth. b) The value was more than $10 \mu \mathrm{g} / \mathrm{ml}$. c) CNS: central nervous system. acids observed were palmitic acid (16:0), palmitoleic acid (16:1), stearic acid (18:0), (E)-9-octadecenoic acid (18:1), $\gamma$-linolenic acid (18:3), octadecatetraenoic acid $(18: 4)$ and icosapentaenoic acid $(20: 5)$. Arachidonic acid $(20: 4)$, the biosynthetic precursor for marine prostanoids, was not detected in the present analysis, although icosapentaenoic acid as a $\mathrm{C}_{20}$ unsaturated fatty acid was observed. Clavulones, the main prostanoids presented in the soft coral Clavularia viridis, was also not detected from the EtOAc-soluble portion of the algal extracts. These findings suggested that clavulones may be produced not by the symbiotic alga, but by the host soft coral itself.

\section{Experimental}

General Experimental Procedures Optical rotations were measured with a JASCO DIP-370 automatic polarimeter. IR spectra were recorded with a Perkin-Elmer FT-IR 1600 spectrophotometer, and visible (VIS) spectra with a JASCO V-520 spectrophotometer. All NMR spectra were recorded with a Bruker DRX-500 $\left({ }^{1} \mathrm{H} ; 500 \mathrm{MHz},{ }^{13} \mathrm{C} ; 125 \mathrm{MHz}\right)$ in $\mathrm{CDCl}_{3} .{ }^{1} \mathrm{H}-{ }^{1} \mathrm{H}$ correlation spectroscopy (COSY), HMQC and HMBC spectra were measured by a Bruker DRX-500 using standard Bruker pulse sequences. Chemical shifts are given on a $\delta(\mathrm{ppm})$ scale with $\mathrm{CHCl}_{3}\left({ }^{1} \mathrm{H} ; 7.26 \mathrm{ppm},{ }^{13} \mathrm{C}\right.$; $77.0 \mathrm{ppm}$ ) as the internal standard (s; singlet, d; doublet, t; triplet, q; quartet, m; multiplet, br; broad). MS were taken with a Micromass Auto Spec spectrometer. Column chromatography was carried out on silica gel 60 (70-230 mesh, Merck) and Sephadex LH-20 (Pharmacia). HPLC was conducted with a YMC-Pack SIL-06 column (silica gel, SH-043-5-06, normal phase). GC was undertaken with a Shimadzu GC-14B instrument equipped with a flame-ionization detector (FID) and a ULBON HR-Thermon 3000B fused SIL capillary column $(25 \mathrm{~m} \times 0.25 \mathrm{~mm}$ i.d., Shinwa Chemical Ind. Ltd.). The injection, oven, and detector temperatures were $250^{\circ} \mathrm{C}, 180^{\circ} \mathrm{C}$, and $250^{\circ} \mathrm{C}$, respectively. The carrier gas was He.

Algal Material The symbiotic alga was isolated from the soft coral Clavularia viridis (order Stolonifera, family Clavularidae), collected from a coral reef of Ishigaki Island, Okinawa Prefecture, Japan, and was identified as an unknown species of dinoflagellate of the genus Symbiodinium (order Dinophyceae, family Gymnodiniales) by the analysis of 18S RNA regions. ${ }^{22,23)} \mathrm{A}$ voucher specimen (strain CV-I1) has been on deposit at Tokyo University of Pharmacy and Life Science, Tokyo, Japan.

Culture Condition $^{22)}$ Symbiodinium sp. was grown in 101 culture bottles with a modified INK medium developed by our group referring to the commercially available medium DAIGO (Nihon Pharmacy) $\left[\mathrm{NaNO}_{3}(2 \mathrm{~g})\right.$, $\mathrm{Na}_{2} \mathrm{HPO}_{4}(14 \mathrm{mg}), \mathrm{K}_{2} \mathrm{HPO}_{4}(50 \mathrm{mg}), \mathrm{NH}_{4} \mathrm{Cl}(26.8 \mathrm{mg}), \mathrm{Na}_{2} \mathrm{EDTA} \cdot 2 \mathrm{H}_{2} \mathrm{O}$ $(372 \mu \mathrm{g})$, FeEDTA $(2.59 \mathrm{mg})$, MnEDTA $(3.32 \mathrm{mg})$, vitamin $\mathrm{B}_{1}(2 \mathrm{mg})$, vita$\min \mathrm{B}_{12}(15 \mu \mathrm{g})$, biotin $(15 \mu \mathrm{g}), \mathrm{MnCl}_{2} \cdot 4 \mathrm{H}_{2} \mathrm{O}(0.9 \mathrm{mg}), \mathrm{ZnSO}_{4} \cdot 7 \mathrm{H}_{2} \mathrm{O}$ $(240 \mu \mathrm{g}), \mathrm{CoSO}_{4} \cdot 7 \mathrm{H}_{2} \mathrm{O}(120 \mu \mathrm{g}), \mathrm{CuSO}_{4} \cdot 5 \mathrm{H}_{2} \mathrm{O}(6 \mu \mathrm{g}), \mathrm{H}_{2} \mathrm{SeO}_{3}(3 \mu \mathrm{g})$, artificial seawater 101$]$ at $23^{\circ} \mathrm{C}$ for $40 \mathrm{~d}$ (a $14: 10$-h light:dark regime, ca. $20 \mu$ mol photons $\mathrm{m}^{-2} \mathrm{~s}^{-1}$ ).

Extraction and Isolation Wet specimens of the cultured alga $(44 \mathrm{~g}$ from 701 of media) were extracted with $\mathrm{MeOH}$. The $\mathrm{MeOH}$ extract $(3.36 \mathrm{~g})$ was partitioned between EtOAc and $\mathrm{H}_{2} \mathrm{O}$. The EtOAc soluble portion $(1.48 \mathrm{~g})$ was chromatographed on a silica gel column. Stepwise elution with hexane, hexane-EtOAc $(3: 1$ and $1: 1)$, EtOAc, and $\mathrm{MeOH}$ gave five fractions (fractions $1-5)$. A portion $(5.9 \mathrm{mg})$ of fraction $1(78 \mathrm{mg}$, eluted with hexane) was treated with $5 \% \mathrm{HCl}-\mathrm{MeOH}(2 \mathrm{ml})$ at $90^{\circ} \mathrm{C}$ for $2 \mathrm{~h}$ to give a mixture of fatty acid methyl esters, which was analyzed by GC. A portion $(36 \mathrm{mg})$ of fraction 3 [116 mg, eluted with hexane-EtOAc $(2: 3)]$ was chromatographed on a Sephadex LH-20 column. Elution with $\mathrm{MeOH}$ gave three fractions, and the second fraction $(16 \mathrm{mg}$ ) was further subjected to HPLC [normal phase, hexane-EtOAc $(2: 3)$ ] to give compound $\mathbf{1}(2.4 \mathrm{mg})$ and $\mathbf{2}$ $(3.8 \mathrm{mg})$, along with peridinin $(3,2.2 \mathrm{mg})$.

Compound 1: Reddish viscous oil. $[\alpha]_{\mathrm{D}}^{25}+63.6^{\circ}\left(c=0.07, \mathrm{CHCl}_{3}\right)$. VIS $\lambda_{\max }(\mathrm{MeOH}) \mathrm{nm}(\varepsilon): 449(57800)$. IR (dry film) $\mathrm{cm}^{-1}: 3458,1929,1732$, 1250. ${ }^{1} \mathrm{H}-$ and ${ }^{13} \mathrm{C}-\mathrm{NMR}$, see Table 1. EI-MS $\mathrm{m} / \mathrm{z}$ : $630[\mathrm{M}]^{+}$. HR-EI-MS $m / z: 630.3528$ (Calcd for $\mathrm{C}_{39} \mathrm{H}_{50} \mathrm{O}_{7}: 630.3557[\mathrm{M}]^{+}$).

Compound 2: Reddish viscous oil. $[\alpha]_{\mathrm{D}}^{25}-69.2^{\circ}\left(c=0.04, \mathrm{CHCl}_{3}\right)$. VIS $\lambda_{\max }(\mathrm{MeOH}) \mathrm{nm}(\varepsilon): 449$ (55800). IR (dry film) $\mathrm{cm}^{-1}: 3454,1929,1732$, 1260. ${ }^{1} \mathrm{H}$ - and ${ }^{13} \mathrm{C}-\mathrm{NMR}$, see Table 1. EI-MS $\mathrm{m} / \mathrm{z}$ : $630[\mathrm{M}]^{+}$. HR-EI-MS $m / z: 630.3547$ (Calcd for $\mathrm{C}_{39} \mathrm{H}_{50} \mathrm{O}_{7}: 630.3557[\mathrm{M}]^{+}$).

Peridinin (3): Reddish viscous oil. ${ }^{1} \mathrm{H}-\mathrm{NMR}\left(500 \mathrm{MHz}, \mathrm{CDCl}_{3}\right) \delta: 0.97$ $(3 \mathrm{H}, \mathrm{s}, \mathrm{H}-17), 1.07$ (3H, s, H-16'), $1.20(3 \mathrm{H}, \mathrm{s}, \mathrm{H}-18), 1.21(3 \mathrm{H}, \mathrm{s}, \mathrm{H}-16)$, $1.26(1 \mathrm{H}, \mathrm{dd}, J=10.8,12.8 \mathrm{~Hz}, \mathrm{H}-2), 1.35\left(3 \mathrm{H}, \mathrm{s}, \mathrm{H}-18^{\prime}\right), 1.38(3 \mathrm{H}, \mathrm{s}, \mathrm{H}-$ 
$\left.17^{\prime}\right), 1.40\left(1 \mathrm{H}, \mathrm{m}, \mathrm{H}-2^{\prime}\right), 1.50\left(1 \mathrm{H}, \mathrm{t}, J=12.8 \mathrm{~Hz}, \mathrm{H}-4^{\prime}\right), 1.62(1 \mathrm{H}, \mathrm{m}, \mathrm{H}-2)$, $1.63(1 \mathrm{H}, \mathrm{m}, \mathrm{H}-4), 1.80\left(3 \mathrm{H}, \mathrm{s}, \mathrm{H}-19^{\prime}\right), 1.99\left(1 \mathrm{H}\right.$, br d, $\left.J=12.8 \mathrm{~Hz}, \mathrm{H}-2^{\prime}\right)$, $2.04(3 \mathrm{H}, \mathrm{s}, \mathrm{OAc}), 2.23$ (3H, s, H-20), 2.28 (1H, br d, $\left.J=12.8 \mathrm{~Hz}, \mathrm{H}-4^{\prime}\right), 2.40$ $(1 \mathrm{H}$, br dd, $J=4.9,14.4 \mathrm{~Hz}, \mathrm{H}-4), 3.91(1 \mathrm{H}, \mathrm{m}, \mathrm{H}-3), 5.38\left(1 \mathrm{H}, \mathrm{m}, \mathrm{H}-3^{\prime}\right)$, $5.73(1 \mathrm{H}, \mathrm{s}, \mathrm{H}-12), 6.05\left(1 \mathrm{H}, \mathrm{s}, \mathrm{H}-8^{\prime}\right), 6.11\left(1 \mathrm{H}, \mathrm{d}, J=11.5 \mathrm{~Hz}, \mathrm{H}-10^{\prime}\right), 6.37$ $(1 \mathrm{H}, \mathrm{d}, J=15.6 \mathrm{~Hz}, \mathrm{H}-8), 6.38\left(1 \mathrm{H}, \mathrm{dd}, J=11.5,14.3 \mathrm{~Hz}, \mathrm{H}-14^{\prime}\right), 6.45(1 \mathrm{H}$, br d, $J=10.8 \mathrm{~Hz}, \mathrm{H}-14), 6.51$ (1H, dd, $J=10.8,14.3 \mathrm{~Hz}, \mathrm{H}-15), 6.61(2 \mathrm{H}$, dd, $\left.J=11.5,14.3 \mathrm{~Hz}, \mathrm{H}-11^{\prime}, \mathrm{H}-15^{\prime}\right), 7.02(1 \mathrm{H}, \mathrm{s}, \mathrm{H}-10), 7.17(1 \mathrm{H}, \mathrm{d}, J=15.6 \mathrm{~Hz}$, $\mathrm{H}-7) .{ }^{13} \mathrm{C}-\mathrm{NMR}\left(125 \mathrm{MHz}, \mathrm{CDCl}_{3}\right) \delta: 14.0\left(\mathrm{CH}_{3}, \mathrm{C}-19^{\prime}\right), 15.4\left(\mathrm{CH}_{3}, \mathrm{C}-20\right)$, $19.9\left(\mathrm{CH}_{3}, \mathrm{C}-18\right), 21.4\left(\mathrm{CH}_{3}, \mathrm{OAc}\right), 24.9\left(\mathrm{CH}_{3}, \mathrm{C}-17\right), 29.2\left(\mathrm{CH}_{3}, \mathrm{C}-17^{\prime}\right)$, $29.5\left(\mathrm{CH}_{3}, \mathrm{C}-16\right), 31.3\left(\mathrm{CH}_{3}, \mathrm{C}-18^{\prime}\right), 32.1\left(\mathrm{CH}_{3}, \mathrm{C}-16^{\prime}\right), 35.3(\mathrm{C}, \mathrm{C}-1), 35.8$ $\left(\mathrm{C}, \mathrm{C}-1^{\prime}\right), 40.9\left(\mathrm{CH}_{2}, \mathrm{C}-4\right), 45.2\left(\mathrm{CH}_{2}, \mathrm{C}-4^{\prime}\right), 45.4\left(\mathrm{CH}_{2}, \mathrm{C}-2^{\prime}\right), 47.1\left(\mathrm{CH}_{2}\right.$, $\mathrm{C}-2), 64.2(\mathrm{CH}, \mathrm{C}-3), 67.5(\mathrm{CH}, \mathrm{C}-5), 67.9\left(\mathrm{CH}, \mathrm{C}-3^{\prime}\right), 70.5(\mathrm{C}, \mathrm{C}-6), 70.7$ (C, C-5'), $103.3\left(\mathrm{CH}, \mathrm{C}-8^{\prime}\right), 117.6\left(\mathrm{C}, \mathrm{C}-6^{\prime}\right), 119.2(\mathrm{CH}, \mathrm{C}-12), 121.8(\mathrm{CH}$, C-8), 124.8 (C, C-9), $128.1\left(\mathrm{CH}, \mathrm{C}-10^{\prime}\right), 128.9\left(\mathrm{CH}, \mathrm{C}-15^{\prime}\right), 131.5(\mathrm{CH}, \mathrm{C}-$ $\left.11^{\prime}\right), 133.0\left(\mathrm{CH}, \mathrm{C}-14^{\prime}\right), 133.6(\mathrm{CH}, \mathrm{C}-7), 133.9\left(\mathrm{C}, \mathrm{C}-9^{\prime}\right), 134.0(\mathrm{CH}, \mathrm{C}-$ 13), 136.3 (CH, C-10), 137.2 (CH, C-15), 138.0 (CH, C-14), 146.8 (C, C11), 168.7 (C, C-19), 170.4 (C, OAc), 202.6 (C, C-7'). These NMR data coincided with those ${ }^{24,25)}$ of all-trans-peridinin.

Acknowledgments The authors thank Dr. Y. Shida at the Tokyo University of Pharmacy and Life Science for measurement of mass spectra.

\section{References and Notes}

1) Kikuchi H., Tsukitani Y., Iguchi K., Yamada Y., Tetrahedron Lett., 23, 5171-5174 (1982).

2) Kikuchi H., Tsukitani Y., Iguchi K., Yamada Y., Tetrahedron Lett., 24, 1549-1552 (1983).

3) Iguchi K., Kaneta S., Mori K., Yamada Y., Honda A., Mori Y., Tetrahedron Lett., 27, 5787-5790 (1985).

4) Nagaoka H., Iguchi K., Miyakoshi T., Yamada N., Yamada Y., Tetrahedron Lett., 27, 223-226 (1986).

5) Honda A., Mori Y., Iguchi K., Yamada Y., Mol. Pharmacol., 32, 530 535 (1987).

6) Bader Th., Yamada Y., Ankel H., Antiviral Res., 16, 341-355 (1991).

7) Corey E. J., Lansbury P. T., Jr., Yamada Y., Tetrahedron Lett., 26 $4171-4174$ (1985)

8) Corey E. J., d'Alarcao M., Matsuda S. P. T., Lansbury P. T., Jr., J. Am. Chem. Soc., 109, 289-290 (1987).

9) Corey E. J., Matsuda S. P. T., Tetrahedron Lett., 28, 4247-4250 (1987).

10) Corey E. J., Matsuda S. P. T., Nagata R., Cleaver M. B., Tetrahedron Lett., 29, 2555-2558 (1988).

11) Strain H. H., Svec W. A., Aizetmüller K., Grandolfo M. C., Katz J. J., Kjøsen H., Norgård S., Liaaen-Jensen S., Haxo F. T., Wegfahrt P., Rapoport H., J. Am. Chem. Soc., 93, 1823-1825 (1971).

12) Strain H. H., Svec W. A., Wegfahrt P., Rapoport H., Haxo F. T., Norgård S., Kjøsen H., Liaaen-Jensen S., Acta Chem. Scand. B, 30,
$109-120(1976)$

13) Johansen J. E., Borch G., Liaaen-Jensen S., Phytochemistry, 19, 441444 (1980).

14) The ${ }^{13} \mathrm{C}$ signal at $\delta 31.3\left(\mathrm{CH}_{3}\right)$ for $\mathbf{1}$ (and 2) was found to be overlapped with two methyl signals by measurement of the signal intensity.

15) Kjøsen H., Norgård S., Liaaen-Jensen S., Svec W. A., Strain H. H., Wegfahrt P., Rapoport H., Haxo F. T., Acta Chem. Scand. B, 30, 157164 (1976).

16) Haugen J. A., Englert G., Aakerman T., Glinz E., Liaaen-Jensen S., Acta Chem. Scand., 48, 769-779 (1994). The NMR data of 1 and 2 cited in this reference were only ${ }^{1} \mathrm{H}-\mathrm{NMR}$ chemical shifts, and ${ }^{1} \mathrm{H}$ coupling constants and ${ }^{13} \mathrm{C}$-NMR data were not described. Although the ${ }^{1} \mathrm{H}-\mathrm{NMR}$ data in the reference were identical with those of $\mathbf{1}$ and $\mathbf{2}$ obtained from the alga, respectively, we conducted a similar acid-catalyzed conversion of peridinin $(3)$ and confirmed that the ${ }^{1} \mathrm{H}$ - and ${ }^{13} \mathrm{C}$ NMR data of $\mathbf{1}$ and $\mathbf{2}$ from the alga are identical with those of the diastereomeric products from $\mathbf{3}$, respectively.

17) In order to confirm compounds $\mathbf{1}$ and $\mathbf{2}$ to be natural products or artificial products from peridinin (3) through the isolation process, the following experiments were conducted. A solution of peridinin (3) was kept at room temperature for $3 \mathrm{~d}$ in the presence of Sephadex LH-20 in $\mathrm{MeOH}$ or silica gel in EtOAc. The formation of $\mathbf{1}$ and $\mathbf{2}$ was not observed. Therefore, compounds $\mathbf{1}$ and $\mathbf{2}$ may be the first fully characterized natural products. A compound having the same gross structure of peridinin-5,8-furanoxide as that of $\mathbf{1}$ and $\mathbf{2}$ was described to be obtained from the dinoflagellate Procentrum lima in the preceding papers for 7th Int. Symp. on Marine Natural Products (Capri, 1992) by Gonzalez and coworkers. However, the stereochemistry as well as the spectral data for the compound were not shown in the preceding paper, and subsequent publication regarding the compound has not been found so far.

18) Nishino H., Satomi Y., Tokuda H., Nishino A., Iwashima A., Tanaka Y., Yamano Y., Shibata Y., Torihara M., Tamai Y., Ito M., J. Kyoto Pref. Univ. Med., 100, 831-835 (1991).

19) Nishino H., Mutation Res., 402, 159-163 (1998).

20) Maoka T., Tsushima M., Nishino H., Chem. Pharm. Bull., 50, 16301633 (2002).

21) Yamori T., Matsunaga A., Sato S., Yamazaki K., Komi A., Ishizu K., Mita I., Edatsugi H., Matsuba Y., Takezawa K., Nakanishi O., Kohno H., Nakajima Y., Komatsu H., Andoh T., Tsuruo T., Cancer Res., 59, 4042-4049 (1999)

22) Fujiwara S., Yasui K., Watanabe K., Wakabayashi T., Iguchi K., Tsuzuki M., Mar. Biotechnol., 5, 1-7 (2003).

23) Rowan R., Powers D. A., Mar. Ecol. Prog. Ser., 71, 65-73 (1991).

24) McLean S., Reynolds W. F., John L. M. D., Tinto W. F., Magn. Reson. Chem., 30, 362-363 (1992).

25) Krane J., Aakermann T., Liaaen-Jensen S., Magn. Reson. Chem., 30, 1169-1177 (1992). 